3. G. Nöbeling, Eine Verschärfung des n-Beinsatzes, Fundamenta Mathematicae, vol. 18 (1932), pp. 23-38.

4. N. E. Rutt, Concerning the cut points of a continuous curve when the arc curve, $a b$, contains exactly $n$ independent arcs, American Journal of Mathematics, vol. 51 (1929), pp. 217-246.

5. G. T. Whyburn, Some properties of continuous curves, this Bulletin, vol. 33 (1927), pp. 305-308. 433.

6. - On the cyclic connectivity theorem, this Bulletin, vol. 37 (1931), pp. 429-

7. L. Zippin, Independent arcs of a continuous curve, Annals of Mathematics, (2), vol. 34 (1933), pp. 95-113.

Purdue University

\title{
INVERSES AND ZERO-DIVISORS
}

\section{REINHOLD BAER}

It may happen that an element in a ring is both a zero-divisor and an inverse, that it possesses a right-inverse though no left-inverse, and that it is neither a zero-divisor nor an inverse. Thus there arises the problem of finding conditions assuring the absence of these paradoxical phenomena; and it is the object of the present note to show that chain conditions on the ideals serve this purpose. At the same time we obtain criteria for the existence of unit-elements.

The following notations shall be used throughout. The element $e$ in the ring $R$ is a left-unit for the element $u$ in $R$, if $e u=u$; and $e$ is a leftunit for $R$, if it is a left-unit for every element in $R$. Right-units are defined in a like manner; and an element is a universal unit for $R$, if it is both a right- and a left-unit for $R$.

The element $u$ is a right-zero-divisor, if there exists an element $v \neq 0$ in $R$ such that $v u=0$; and $u$ is a right-inverse in $R$, if there exists an element $w$ in $R$ such that $w u$ is a left-unit for $u$ and a right-unit for $R$. Left-zero-divisors and left-inverses are defined in a like manner. Note that 0 is a zero-divisor, since we assume that the ring $R$ is different from 0 .

$L(u)$ denotes the set of all the elements $x$ in $R$ which satisfy $x u=0$; clearly $L(u)$ is a left-ideal in the ring $R$ and every left-ideal of the form $L(u)$ shall be termed a zero-dividing left-ideal. Principal leftideals ${ }^{1}$ are the ideals of the form $R v$ for $v$ in $R$ and the ideals $v R$ are the principal right-ideals.

Presented to the Society December 31,1941; received by the editors September 19, 1941, and, in revised form, October 13, 1941.

${ }^{1}$ This is a slight change from the customary terminology. 
LEMma 1. If vu is a left-unit for $u$ and a right-unit for $R$, then $u v=v u$, $R v=R$ and $L(u)=0$ are equivalent properties.

Proof. If $u v=v u$, then $R=R v u=R u v \leqq R v \leqq R$, or $R=R v$. If $R=R v$, and if $x u=0$, then there exists an element $y$ in $R$ such that $x=y v$; and $L(u)=0$ is a consequence of $y=y v u=x u=0$. If $L(u)=0$ then $(v u-u v) u=v u u-u v u=u-u=0$ implies that $v u-u v$ is in $L(u)$ and that therefore $v u=u v$.

EXAMPLE 1. Let $G$ be the additive group of all the countably infinite sequences of numbers from the commutative field $F$, considered as an abelian operator group over $F$; and denote by $R$ the ring of all the $F$-admissible automorphisms of $G$ (that is, of all the linear transformations of $G$ over $F$ ). Elements $u, v, w$ in $R$ are defined by

$$
\begin{gathered}
\left(a_{1}, \cdots\right)^{u}=\left(0, a_{1}, \cdots\right), \quad\left(a_{1}, \cdots\right)^{v}=\left(a_{1}, 0, \cdots\right), \\
\left(a_{1}, \cdots\right)^{w}=\left(a_{2}, a_{3}, \cdots\right)
\end{gathered}
$$

and they satisfy $u v=0, u w=1, w u \neq 1$. Thus a left-inverse may be a left-zero-divisor.

Lemma 2. Each of the following properties of the ring $R$ implies all the others.

(i) There exists a universal unit in $R$.

(ii) There exists a right-unit for $R$, and $x=0$ is a consequence of $R x=0$.

(iii) There exists one and only one right-unit for $R$.

(iv) There exist a right-unit for $R$ and a left-unit for $R$.

Proof. (ii) is an obvious consequence of (i). If (ii) holds, and if $u$ and $v$ are right-units for $R$, then $x(u-v)=x u-x v=0$ for every element $x$ in $R$; thus $u=v$ and (iii) is an implication of (ii). If (iii) holds, if $e$ is the uniquely determined right-unit for $R$, and if $x$ and $y$ are elements in $R$, then $x(e+y-e y)=x e+x y-x e y=x+x y-x y=x$ for every $x$ in $R$. Thus $e+y-e y$ is a right-unit for $R$; and the equality of all right-units implies that $y=e y$, that is, that $e$ is also a left-unit for $R$; and (iv) is therefore a consequence of (iii). If (iv) holds, then there exist a right-unit $u$ and a left-unit $v$ for $R$; and (i) may be inferred from $u=v u=v$.

The importance of condition (ii) may be seen from the fact that the elements $x$ in $R$ which satisfy $R x=0$ form a two-sided ideal $T$ whose square is 0 .

LEMMA 3. If the minimum condition is satisfied by the principal leftideals in the ring $R$, if $J$ is a principal left-ideal in $R$ and if $J v=R$ for $v$ an element in $R$, then $J=R$. 
REMARK. Omitting the word "principal" throughout we obtain a true statement that may be proved in a similar fashion.

Proof. If $R=J v$ for $J$ a principal left-ideal and $v$ an element in $R$, then there exists a smallest principal left-ideal $S=R s$ such that $R=S t$ for some $t$ in $R$. Hence $s=r s t$ for $r$ in $R$. Since $R r s \leqq R s=S$, and since $(R r s) t^{2}=R(r s t) t=R s t=S t=R$, it follows from our choice of $S$ that $R r s=S$; and hence $S=R s=R r s t=S t=R$, a fact which proves our contention.

THEOREM 1. The element $z$ in the ring $R$ with minimum condition for principal left-ideals is a right-inverse in $R$ if, and only if, $z$ is not a right-zero-divisor in $R$.

Proof. Assume first the existence of an element $v$ in $R$ such that $v z$ is a left-unit for $z$ and a right-unit for $R$. Then $J=R v$ is a principal left-ideal and it follows from Lemma 3 and the equation $J z=R v z=R$ that $R=J=R v$; and hence it follows from Lemma 1 that $z$ is not a right-zero-divisor.

If there exist elements in $R$ which are not right-zero-divisors, then among these elements there is one, say $v$, with minimal (principal leftideal) $R v$. Since $x v^{2}=0$ implies $x v=0$, and since this implies $x=0$, it follows that $v^{2}$ is not a right-zero-divisor. Thus $R v^{2} \leqq R v$ and the minimum property of $R v$ imply $R v^{2}=R v$. Hence for every element $r$ in $R$ there exists an element $r^{\prime}$ in $R$ such that $r v=r^{\prime} v^{2}$ or $\left(r-r^{\prime} v\right) v=0$. Since $v$ is not a right-zero-divisor, $r-r^{\prime} v=0$ or $r=r^{\prime} v$. Thus we have shown that $R=R v$; and this fact makes it evident that $R=R z$ for every $z$ which is not a right-zero-divisor in $R$.

If the element $z$ in $R$ is not a right-zero-divisor in $R$, then $R=R z$; and there exists therefore one (and only one) element $e$ in $R$ such that $e z=z$. Since $(r-r e) z=r z-r z=0$ for every $r$ in $R$, it follows that $e$ is both a left-unit for $z$ and a right-unit for $R$. Since the existence of an element $w$ satisfying $w z=e$ is a consequence of $R=R z$, it has been shown that $z$ is a right-inverse in $R$.

An immediate consequence of this theorem is the following fact.

COROLlary. The ring $R$ with minimum condition for principal leftideals contains a right-unit for $R$ if (and only if) at least one element in $R$ is not a right-zero-divisor.

The impossibility of substituting the maximum condition for the minimum condition in Theorem 1 and its corollary may be seen from the example of the even rational integers. The example of the ring of all the matrices 


$$
\left(\begin{array}{ll}
0 & a \\
0 & b
\end{array}\right)
$$

with coefficients from some commutative field shows that the conditions of the corollary are not sufficient for the existence of a universal unit.

THEOREM 2. If there exists a universal unit 1 in the ring $R$, if the minimum condition is satisfied by both the principal right-and the principal left-ideals in $R$, then each of the following properties of the element $z$ in $R$ implies all the others:

(a) $z$ is not a right-zero-divisor.

(b) $z$ is a right-inverse.

(c) $z$ is not a left-zero-divisor.

(d) $z$ is a left-inverse.

That (b) implies (c) and that (d) implies (a) are obvious consequences of Lemma 1 ; and that (a) implies (b) and that (c) implies (d) may be inferred from Theorem 1 and Lemma 2.

The impossibility of substituting maximum conditions for the minimum conditions in this theorem may be seen from the example of the ring of all the rational integers.

THEOREM 3. If the minimum condition is satisfied by the principal left-ideals and by the zero-dividing left-ideals in the ring $R$, then $R=R z$ is a necessary and sufficient condition for $z$ to be a right-inverse in $R$.

PRoof. If $z$ is a right-inverse in $R$, then $v z$ is for some $v$ in $R$ a rightunit for $R$ and $R=R v z \leqq R z \leqq R$ or $R=R z$.

If, conversely, $R=R z$, then $z=e z$ for some $e$ in $R$. The principal left-ideal $J=R e$ satisfies $J z=R e z=R z=R$. Hence it follows from Lemma 3 that $R=R e$ for every left-unit $e$ of the element $z$.

Among the left-units for $z$ there exists one, say $f$, with minimal $L(f)$. Then it is a consequence of $R=R f$ that $f=g f$ for some $g$ in $R$. Since $g z=g(f z)=(g f) z=f z=z$, and since $x g=0$ implies $0=x g f=x f$, it follows that $g$ is a left-unit for $z$ and that $L(g) \leqq L(f)$. Hence $L(g)=L(f)$ is a consequence of our choice of $f$. Since $\left(g^{2}-g\right) f=f-f=0$, the element $g^{2}-g$ is in $L(f)=L(g)$ so that $\left(g^{2}-g\right) g=0$ or $g^{3}=g^{2}$. Consequently the element $e=g^{2}$ satisfies $e^{2}=g^{4}=g^{3}=g^{2}=e$ and $e z=g^{2} z=z$, that is, the element $e$ is an idempotent and a left-unit for $z$. Thus $e$ is an idempotent satisfying $R=R e$ (as has been shown in the second paragraph of the proof); and the left-unit $e$ for $z$ is therefore a right-unit for $R$. Finally there exists an element $w$ in $R$ such that $w z=e$, since $R=R z$; and this completes the proof of the fact that $z$ is a right-inverse in $R$. 
The following fact is an immediate consequence of Theorem 3.

Corollary. The ring $R$ with minimum condition for principal and for zero-dividing left-ideals contains a right-unit for $R$ if, and only if, $R$ is a principal left-ideal in $R$.

As an application of the preceding results we prove the following statement.

The ring $R$ is a (not necessarily commutative) field if, and only if,

(a) 0 is the only right-zero-divisor in $R$,

(b) the minimum condition is satisfied by the principal left-ideals in $R$.

It is obvious that the two conditions are necessary and that neither of them could be omitted. If the conditions (a) and (b) are satisfied by $R$, then every element $z \neq 0$ in $R$ is a right-inverse by Theorem 1 . Since $R$ contains therefore a right-unit for $R$, it follows from Lemma 2 that $R$ contains a universal unit 1 ; and it follows from Lemma 1 that every element $z \neq 0$ in $R$ is both a right- and a left-inverse. Hence $R$ is a field.

LEMMA $4 .^{2}$ If the maximum condition is satisfied by the zero-dividing left-ideals in the ring $R$, if the left-ideal $J$ and the element $z$ in $R$ satisfy $J z=R$, then $L(z)=0$ and $J=R$.

Proof. If $R=J z$ for some left-ideal $J$ and some element $z$ in $R$, then among these elements $z$ there exists one, say $w$, with maximal $L(w)$. If $W$ is some left-ideal such that $R=W w$, then $R=W w \leqq R w$ $=W w^{2} \leqq R$ or $R=W w^{2}$. Hence it follows from our choice of $w$ and the obvious inequality $L(w) \leqq L\left(w^{2}\right)$ that $L(w)=L\left(w^{2}\right)$. Every element in $L(w)$ has the form $x=y w$ for $y$ in $W$, since $R=W w$. If $y w$ is in $L(w)$, then $y$ is in $L\left(w^{2}\right)=L(w), y w=0$ and consequently $L(w)=0$. Thus we have shown that $L(z)=0$ whenever $R=J z$ for some left-ideal $J$ in $R$.

If $J z=R$, then for every element $r$ in $R$ there exists an element $j$ in the left-ideal $J$ such that $j z=r z$. This implies $j=r$, since $z$ is not a right-zero-divisor. This completes the proof of the fact that $J=R$ and $L(z)=0$ are consequences of $J z=R$.

Theorem 4. Suppose that the maximum condition is satisfied by the zero-dividing left-ideals in the ring $R$.

(a) $u v$ is a right-unit for $R$ if, and only if, vu is a right-unit for $R$.

(b) The right-unit vu for $R$ is a left-unit for $u$ if, and only if, $u v=v u$.

(c) $R$ contains a right-unit (for $\mathrm{R}$ ) if, and only if, $R$ is a principal left-ideal (in $R$ ).

Proof. If $v u$ is a right-unit for $R$, then $R=R v u$; and it follows from

${ }^{2}$ Cf. Lemma 3. 
Lemma 4 that $L(u)=0, R v=R, L(v)=0, R u=R$. If $r$ is any element in $R$, then $(r-r u v) u=r u-r u(v u)=r u-r u=0$. Since $L(u)=0$, we find that $r=r u v$ for every $r$ in $R$. This proves (a); and (b) may be obtained as an immediate consequence of Lemma 1 . If $R=R z$ for some $z$ in $R$, then it follows from Lemma 4 that $L(z)=0$. Furthermore, there exists an element $e$ in $R=R z$ such that $z=e z$. If $r$ is any element in $R$, then $(r-r e) z=r z-r z=0$. The element $e$ is a right-unit for $R$, since $L(z)=0$ implies $r=r e$ for every $r$. This completes the proof of the theorem.

THEOREM 5. If there exists a universal unit 1 in $R$, if the minimum condition for principal left-ideals or the maximum condition for zero-dividing left-ideals is satisfied in $R$, then the relations $u v=1$ and $v u=1$ imply each other.

Proof. If the maximum condition is satisfied by the zero-dividing left-ideals in $R$, then our contention is an immediate consequence of Theorem 4, (a) and Lemma 2. If the minimum condition is satisfied by the principal left-ideals in $R$, then $u v=1$ implies $L(u)=0$. By Theorem 1 there exists an element $w$ such that $w u=1$. But $w=w u v=v$ so that $v u=w u=1$.

The impossibility of omitting the chain conditions in Theorem 5 is a consequence of Example 1.

The following special case of Theorem 5 may be worth mentioning, since it solves a problem of importance in the theory of matrices. ${ }^{3}$ If $R$ is a ring with universal unit 1 , then denote by $R_{n}$ the ring of all square matrices with $n$ rows and $n$ columns whose coefficients are in $R$. If the maximum (minimum) condition is satisfied by the left-ideals in $R$, then the same condition holds in $R_{n}$; and hence it is a consequence of Theorem 5 that the matrices $A$ and $B$ in $R_{n}$ satisfy either $A B=1$ and $B A=1$ or neither of these relations; in particular it is impossible that a matrix in $R_{n}$ is both a zero-divisor and an inverse in $R_{n}$.

A right- or left-ideal $J$ in the $\operatorname{ring} R$ is termed nilpotent, if $J^{i}=0$ for some positive integer $i$. The sum $P=P(R)$ of all the nilpotent right- and left-ideals in $R$ is a two-sided ideal in $R$ which is called the radical of $R$. Hopkins ${ }^{4}$ has shown that the radical itself is nilpotent, if the minimum condition is satisfied by the left-ideals in $R$.

TheOREM 6. There exists a left-unit ${ }^{5}$ for the ring $R$ with minimum condition for left-ideals if, and only if,

${ }^{3}$ Cf., for example, B. L. van der Waerden, Moderne Algebra, vol. 2, Berlin, 1927, pp. $114-115$.

${ }^{4}$ C. Hopkins, Annals of Mathematics, (2), vol. 40 (1939), pp. 712-730; Theorem 1.4, p. 714 .

${ }^{5}$ Note that the preceding criteria assured the existence of right-units for the ring $R$. 
(i) $R x=0$ implies $x=0$,

(ii) $P^{i} R x=x P=0$ implies $P^{i} x=0$ for $0<i<m$ (where $m$ is the smallest positive integer such that $P^{m}=0$ ).

Proof. If $R$ contains a left-unit for every element then $x$ is always an element of $R x$; and this shows the necessity of conditions (i) and (ii).

Suppose that conditions (i) and (ii) are satisfied by the ring $R$. From the choice of the integer $m$ it follows that $0=P^{m}<P^{m-1}$ (using the notation $P^{0}=R$ ). If $x$ is an element not 0 in $P^{m-1}$, then $P x=0$ so that $P<R$ is a consequence of condition (i). Hence ${ }^{6}$ there exists in $R$ an idempotent $e \neq 0$ such that $R=R e+P$.

Denote by $T$ the set of all the elements $x$ in $R$ which satisfy: $x P=0$; and denote by $S(i)$ (for positive $i$ ) the set of all those elements in $T$ which satisfy: $P^{i} x=0$. Clearly $T$ and therefore every $S(i)$ is a twosided ideal in $R$. We are going to prove by complete induction that $e S(i)=S(i)$. This is true for $i=1$, since it follows from $R x=R e x+P x$ and condition (i) that $x=0$ is the only element $x$ in $S(1)$ which satisfies $e x=0$. Suppose now that $S(i)=e S(i)$ and that the element $t$ in $S(i+1)$ satisfies $e t=0$. Then $R t=R e t+P t=P t \leqq P S(i+1) \leqq S(i)$, $P^{i} R t \leqq P^{i} S(i)=0$; and it follows from condition (ii) that $P^{i} t=0$. The element $t$ is therefore an element in $S(i)=e S(i)$, that is, $t=e t=0$ or $S(i+1)=e S(i+1)$; and this completes the induction. In particular it follows that $T=e T$, since $T=S(m)$ is a consequence of $P^{m}=0$.

Denote by $T(i)$ the set of all the elements $x$ in $R$ which satisfy $x P^{i}=0$. Every $T(i)$ is a two-sided ideal in $R$. We are going to prove by complete induction that $T(i)=e T(i)$. This is true for $i=1$, since $T=T(1)$. Suppose that $T(i)=e T(i)$ and that the element $v$ in $T(i+1)$ satisfies $e v=0$. Then $v P=0$, since $v P \leqq T(i)=e T(i)$ and $e v=0$. Thus $v$ is an element in $T=e T$ and $v=e v=0$. This shows that $T(i+1)$ $=e T(i+1) ;$ and this completes the induction. Since $P^{m}=0$ implies $T(m)=R$, we have proved in particular that $R=e R$; and the idempotent $e$ is therefore a left-unit for $R$.

We note that we proved the following fact. If the conditions (i) and (ii) of Theorem 6 are satisfied by the ring $R$, if the radical of $R$ is nilpotent, and if the idempotent $e$ in $R$ satisfies $R=R e+P$, then $e$ is a left-unit for $R$; and we mention that the minimum condition for zerodividing left-ideals would be sufficient to assure the existence of such an idempotent $\boldsymbol{e}$.

One verifies readily that the conditions (i) and (ii) of Theorem 6 are consequences of each of the following (not necessary) conditions

${ }^{6}$ Cf. Hopkins, loc. cit., Theorems 4.1, 4.2, p. 721. 
(a) $P=0$; (b) condition (i), and $x P=0$ implies $P x=0$; (c) condition (i), and $R P^{i} \leqq P^{i} R$.

That condition (ii) is indispensable for the validity of Theorem 6 may be seen from the following example. Denote by $R$ the ring of all the matrices of the form

$$
\left(\begin{array}{lll}
a & 0 & 0 \\
b & 0 & 0 \\
c & d & e
\end{array}\right)
$$

with coefficients from some (commutative) field $F$. It is readily verified that $R x=0$ implies $x=0$; that $y R=0$ implies $y=0$; and that this ring $R$ does not contain a universal unit 1 . We note that $R$ would be a finite ring, if $F$ were a finite field.

COROLlARY. There exists a left-unit for the ring $R$ with minimum condition for left-ideals if (and only if) there exists a left-unit for every element in $R$.

Proof. The existence of a left-unit for the element $x$ in $R$ is equivalent to the fact that $x$ is an element in the left-ideal $R x$. This shows that the conditions (i), (ii) of Theorem 6 are consequences of the condition of the corollary.

THEOREM 7. There exists a universal unit in the ring $R$ with minimum condition for left-ideals if, and only if,

(1) $R x=0$ implies $x=0$,

(2) $y R=0$ implies $y=0$,

(3) $R P=P R$.

Proof. The necessity of the conditions is obvious. If the conditions are satisfied by $R$, then it follows from (1), (3) that conditions (i), (ii) of Theorem 6 are satisfied. Hence there exists a left-unit $e$ for $R$; and it follows from Lemma 2 and condition (2) that $e$ is a universal unit for $R$.

COROLlaRy. There exists a universal unit in the commutative ring $R$ with minimum condition for ideals if, and only if, $R x=0$ implies $x=0$.

This is an immediate consequence of Theorem 7 .

The impossibility of substituting the maximum condition for the minimum condition in Theorems 6 or 7 may be seen from the example of the even rational integers.

Comparing the results of this investigation one sees that minimum conditions are more powerful than maximum conditions. This phe- 
nomenon is closely connected with Hopkins' theorem : ${ }^{7}$ If the ring $R$ contains a left-unit or a right-unit for $R$, then the maximum condition for left-ideals in $R$ is a consequence of the minimum condition for leftideals in $R$. We could not make use of this theorem, since we usually considered rings with minimum (maximum) condition for principal or zero-dividing left-ideals. On the other hand it is possible to improve slightly the theorem of Hopkins by applying our criteria for the existence of units.

It is well known that the commutative law of addition is a consequence of the customary postulates for a field, but not of the analogous postulates for a ring. To investigate this situation we consider a nonvacuous set $R$ of elements which are connected by two operations: addition $u+v$ and multiplication $u v$, subject to the following rules:

I. $R$ is a group under addition.

II. The product $u v$ of the elements $u$ and $v$ in $R$ is a uniquely determined element in $R$.

III. $u(v+w)=u v+u w,(u+v) w=u w+v w$.

We denote by $R^{2}$ the subgroup of the additive group $R$ which is generated by all the products $u v$ and by $C=C(R)$ the commutator subgroup of the additive group $R$. Both $R^{2}$ and $C$ are two-sided ideals in $R$.

The addition is commutative in $R^{2}$, and $C R=R C=0$.

Proof. If $a, b, c, d$ are elements in $R$, then it follows from the distributive laws that

$$
a d+a b+c d+c b=(a+c)(d+b)=a d+c d+a b+c b ;
$$

and hence it follows from the cancellation law of addition that

$$
a b+c d=c d+a b .
$$

Our contention is an immediate consequence of this equality.

It is now obvious that each of the following two conditions is sufficient to assure commutativity of addition.

(a) $R=R^{2}$.

(b) 0 is the only element in $R$ which satisfies both $R x=0$ and $x R=0$.

We note that each of these two conditions is necessary, but not sufficient for the existence of a universal unit in $R$.

UNIVERSITY OF ILLINOIS

${ }^{7}$ Hopkins, loc. cit., Theorem 6.7, p. 728. 